\title{
Electrophysiologic Mechanisms of Functional Bundle Branch Block at Onset of Induced Orthodromic Tachycardia in the Wolff-Parkinson-White Syndrome
}

\author{
Role of Stimulation Method
}

\author{
Michael H. Lehmann, Stephen Denker, Rehan Mahmud, Patrick Tchou, John Dongas, and Masood Akhtar \\ Natalie and Norman Soref and Family Electrophysiology Laboratory, University of Wisconsin-Milwaukee Clinical Campus, Mount Sinai \\ Medical Center, Milwaukee, Wisconsin 53233
}

\begin{abstract}
The mechanisms of aberrant conduction at the onset of induced orthodromic tachycardia in the Wolff-Parkinson-White syndrome were analyzed in $\mathbf{2 0}$ consecutive patients in whom this tachycardia was initiated by the atrial $\left(A_{2}\right)$ and/or right ventricular $\left(V_{2}\right)$ extrastimulus techniques. Of 13 patients in whom orthodromic tachycardia was induced by the $A_{2}$ method, functional right bundle branch block occurred at tachycardia onset in four (31\%) and left bundle aberrancy in two (15\%), one of whom also manifested right bundle aberrancy. The occurrence of bundle branch block at the onset of tachycardia was linked to aberrant conduction of the initiating $A_{2}$ impulse which, in turn, was associated with attainment of relatively short $\mathrm{His}_{1} \mathrm{His}_{2}$ intervals within the tachycardia initiation zone. Aberrant conduction of $A_{2}$ was also more common in patients without manifest preexcitation. In contrast, of 14 patients in whom orthodromic tachycardia was induced by the $V_{2}$ method, left bundle aberrancy occurred at the onset of tachycardia in 11 (79\%), one of whom manifested right bundle branch block as well. Left bundle aberrancy was more likely to occur when the interval from the initiating $V_{2}$ (or macro-reentrant $V_{3}$ ) impulse to the first anterograde His deflection was $<300 \mathrm{~ms}$. This suggests that left bundle aberrancy at the onset of orthodromic tachycardia induced by the $V_{2}$ method results from concealed retrograde penetration of the HisPurkinje system, with the left bundle being last to recover. Our findings provide the conceptual basis for a physiologic approach to the deliberate induction of specific types of aberrant conduction at onset of orthodromic tachycardia in patients with Wolff-Parkinson-White syndrome.
\end{abstract}

\section{Introduction}

Accessory pathway localization is an important objective during electrophysiologic assessment of patients with Wolff-ParkinsonWhite syndrome. ${ }^{1}$ Of special value in this regard is the quantitation of changes in ventriculoatrial conduction time attending

Address reprint requests to Dr. Lehmann at his current address: Division of Cardiology, Harper Hospital, 3990 John R, Detroit, Michigan 48201.

Received for publication 15 February 1985 and in revised form 22 April 1985.

1. For the purpose of this article, we will apply the eponym "WolffParkinson-White syndrome" to all patients with accessory atrioventricular pathways, whether or not there is manifest ventricular preexcitation.

J. Clin. Invest.

(C) The American Society for Clinical Investigation, Inc. 0021-9738/85/10/1566/09 \$1.00

Volume 76, October 1985, 1566-1574 the occurrence of particular types of functional $(\mathrm{fx})^{2}$ bundle branch block (BBB) during orthodromic tachycardia (OT) (1, 2). Unfortunately, little is known regarding the means by which one may deliberately induce specific $f x$ BBB patterns during OT using programmed stimulation, other than exhaustive trial and error (2), Moreover, the relationship (if any) between particular mechanisms of OT initiation and the occurrence of aberrant conduction during OT has not been systematically investigated.

To gain insight into this problem, we elected to focus upon the determinants of aberrant conduction at onset of OT initiated by either atrial or right ventricular extrastimulation $\left(A_{2}\right.$ and $V_{2}$ methods, respectively). The effects of these induction techniques, in particular, can be precisely related to the events at onset of induced OT. Moreover, these two stimulation methods initiate OT by entirely different mechanisms: induction of OT by $\mathrm{A}_{2}$ requires anterograde block in the accessory pathway with effective propagation along the atrioventricular node and His-Purkinje system (3). In contrast, $\mathrm{V}_{2}$ generally starts OT by inducing bilateral retrograde infra-His block (4) (causing concealed conduction in the His-Purkinje system [5]) with concomitant retrograde atrial activation occurring via the bypass tract.

We conjectured that, in view of these disparate modes of input to the normal pathway during OT induction, refractoriness of the His-Purkinje system should be differentially encountered and manifested with the $A_{2}$ and $V_{2}$ methods. This hypothesis was evaluated using a beat-by-beat analysis in 20 consecutive patients with accessory pathways and OT induced by the $A_{2}$ and/or $V_{2}$ techniques. Our findings suggest that a rational physiologically oriented approach to the deliberate induction of $\mathrm{fx}$ $\mathrm{BBB}$ at OT onset is indeed feasible.

\section{Methods}

Electrophysiologic studies were performed in the nonsedated, postabsorptive state after informed consent was obtained and after discontinuation of antiarrhythmic drugs for at least $48 \mathrm{~h}$. Under local anesthesia, three or more multipolar electrode catheters were introduced percutaneously via peripheral veins and, with fluoroscopic guidance, positioned in the high right atrium, coronary sinus, right ventricular apex (or outflow tract), and the region of the His (and right) bundle. The catheters were used to record local bipolar intracardiac electrograms and to perform programmed stimulation. All intracardiac electrograms, surface electrocardiographic leads I, II, and $V_{1}$, and time lines were simultaneously displayed on a multichannel oscilloscope and recorded on an FM tape

2. Abbreviations used in this paper: $\mathrm{A}_{2}$ method, atrial extrastimulus method; AH, atrio-His; BBB, bundle branch block; fx, functional; $\mathrm{HV}$, His-ventricular; LBBB, left bundle branch block; OT, orthodromic tachycardia; $R B B B$, right bundle branch block; $V_{2}$ method, right ventricular extrastimulus method; VA, ventriculoatrial; $\mathrm{VH}$, ventricularHis. 
recorder for subsequent reproduction at a paper speed of $100 \mathrm{~mm} / \mathrm{s}$ (Electronics for Medicine model VR-16 [Pleasantville, NY]). Intracardiac stimulation was performed with a digital stimulator (Bloom Associates, Reading, PA) capable of delivering rectangular impulses of 5-V amplitude and 2-ms duration.

The stimulation protocol included incremental atrial and ventricular pacing as well as $A_{2}$ and $V_{2}$ methods (at one or more basic cycle lengths) to assess anterograde and retrograde conduction and refractory periods, and to induce OT as previously described (6). Accessory pathway localization was accomplished using standard criteria (7), including quantitation of the change in ventriculoatrial (VA) interval (measured on the His bundle electrogram) accompanying the occurrence of sustained ( $\geq$ three beats of) fx BBB during OT (2).

Patient characteristics. Base-line clinical and electrophysiologic data of the 20 patients studied are indicated in Table I. There were 11 males and 9 females ranging in age from 8 to $59 \mathrm{yr}$. No patients had evidence of structural heart disease. Accessory pathways were left-sided in 14 patients, paraseptal in 5, and right-sided in 1. Manifest preexcitation during sinus rhythm (or atrial pacing) was present in 14 with the remainder demonstrating accessory pathway conduction only in the retrograde direction. The OT cycle lengths ranged from 250 to $410 \mathrm{~ms}$ with a mean of $328 \pm 48 \mathrm{~ms}$. During OT all patients were capable of conducting with narrow QRS complexes ( $\leq 90 \mathrm{~ms}$ in duration) associated with His-ventricular (HV) intervals ranging from 35 to $60 \mathrm{~ms}$.

Definitions. OT onset refers to the first induced ventricular complex (regardless of stimulation technique) resulting exclusively from reentrant excitation of the atria and His bundle (anterogradely). It follows from this working definition that when OT is induced by the $A_{2}$ method, the ventricular response to the paced $A_{2}$ precedes the first OT complex.

Complete right $(\mathrm{R})$ and left $(\mathrm{L}) \mathrm{BBB}$ were defined according to accepted electrocardiograph (ECG) criteria (8).

Incomplete LBBB was considered present when $(a) \mathrm{QRS}$ duration was prolonged (versus narrow-QRS OT) to a value $<120 \mathrm{~ms}$ with loss of septal $\mathrm{Q}$ wave in lead I, and/or $(b)$ the frontal axis was shifted leftward (to -30 or beyond often with a deeper $S$ wave in $V_{1}$ ) or, rarely, rightward (to +90 or beyond but without evidence of terminal delay). This definition was used to include all forms of manifest left bundle conduction delay short of complete LBBB.

Incomplete RBBB was defined by the appearance of terminal rightward conduction delay with $\mathrm{QRS}$ prolongation to a value $<120 \mathrm{~ms}$.

When OT was induced by the $\mathrm{V}_{2}$ method, differentiation of anterograde from retrograde origin of the (emergent) His deflection following $\mathrm{V}_{2}$ was accomplished using previously published criteria (4). It was possible, therefore, to distinguish also between fx complete LBBB (at OT onset) and macro-reentrant $V_{3}$ induced by $V_{2}(9)$.

VH interval (in cases of OT induced with the $V_{2}$ method) was measured from the onset of the initiating $V_{2}$ (or $V_{3}$ ) electrogram to the onset of the anterograde His deflection immediately preceding the first beat of OT. This parameter (which equals VA + atrio-His[AH] intervals) was considered an estimate of the time elapsed from concealed retrograde penetration of the His-Purkinje system by the initiating right ventricular impulse to the onset of initial reentrant anterograde His activation.

Statistical analysis. Measured values are expressed as mean \pm standard deviation. The unpaired $t$ test was utilized to compare differences in means. Statistical significance was defined as $P<0.05$.

\section{Results}

For reasons stated above, only findings pertinent to OT initiation by the $A_{2}$ or $V_{2}$ methods form the basis of this report. Moreover, for each patient, only data obtained at the basic cycle length associated with the widest aberrant complex at OT onset are presented so as to simplify analysis of the results.

Table I. Base-line Clinical and Electrophysiologic Data

\begin{tabular}{|c|c|c|c|c|c|c|c|c|c|c|c|}
\hline \multirow{2}{*}{$\begin{array}{l}\text { Patient } \\
\text { no. }\end{array}$} & \multirow[b]{2}{*}{ Age $(y r) / \operatorname{sex}$} & \multirow{2}{*}{$\begin{array}{l}\text { Location of } \\
\text { AP }\end{array}$} & \multirow{2}{*}{$\begin{array}{l}\text { Manifest } \\
\text { preexcitation }\end{array}$} & \multicolumn{6}{|c|}{ Characteristics of OT } & \multicolumn{2}{|c|}{$\begin{array}{l}\text { Induction } \\
\text { method }\end{array}$} \\
\hline & & & & $\mathrm{CL}$ & QRS & Axis & $\mathrm{AH}$ & HV & VA & $\mathbf{A}_{2}$ & $\mathrm{~V}_{2}$ \\
\hline & & & & $m s$ & $m s$ & & $m s$ & $m s$ & $m s$ & & \\
\hline 1 & $30 / \mathrm{F}$ & Left FW & + & 370 & 80 & N1 & 210 & 50 & 110 & + & - \\
\hline 2 & $17 / \mathrm{F}$ & Left FW & - & 410 & 90 & N1 & 235 & 50 & 125 & + & - \\
\hline 3 & $32 / \mathrm{M}$ & Left FW & + & 385 & 80 & N1 & 210 & 55 & 120 & + & - \\
\hline 4 & $59 / \mathrm{M}$ & Left FW & + & 340 & 70 & N1 & 135 & 55 & 150 & + & - \\
\hline 5 & $8 / F$ & Right FW & + & 250 & 80 & N1 & 85 & 40 & 125 & + & - \\
\hline 6 & $42 / \mathrm{F}$ & Left FW & - & 360 & 80 & N1 & 200 & 40 & 120 & + & - \\
\hline 7 & $16 / \mathrm{M}$ & Left FW & - & 310 & 90 & $\mathrm{Nl}$ & 145 & 45 & 120 & + & + \\
\hline 8 & $43 / \mathrm{M}$ & Paraseptal & + & 350 & 70 & N1 & 185 & 50 & 115 & + & + \\
\hline 9 & $17 / \mathrm{M}$ & Left FW & - & 340 & 90 & $\mathrm{~N} 1$ & 150 & 50 & 140 & + & + \\
\hline 10 & $43 / F$ & Paraseptal & + & 290 & 80 & $\mathrm{~N} 1$ & 155 & 35 & 100 & + & + \\
\hline 11 & $18 / \mathrm{M}$ & Paraseptal & - & 260 & 90 & $\mathrm{~N} 1$ & 105 & 45 & 110 & + & + \\
\hline 12 & $30 / \mathrm{F}$ & Left FW & + & 270 & 80 & $\mathrm{~N} 1$ & 110 & 40 & 120 & + & + \\
\hline 13 & $21 / F$ & Left FW & + & 365 & 90 & N1 & 205 & 40 & 120 & + & + \\
\hline 14 & $40 / \mathrm{F}$ & Paraseptal & + & 260 & 80 & N1 & 130 & 40 & 90 & - & + \\
\hline 15 & $29 / \mathrm{F}$ & Paraseptal & + & 290 & 80 & N1 & 140 & 50 & 100 & - & + \\
\hline 16 & $56 / \mathrm{M}$ & Left FW & + & 390 & 80 & N1 & 225 & 45 & 120 & - & + \\
\hline 17 & $20 / \mathrm{M}$ & Left FW & + & 380 & 80 & N1 & 210 & 50 & 120 & - & + \\
\hline 18 & $44 / M$ & Left FW & - & 320 & 90 & $\mathrm{~N} 1$ & 140 & 50 & 130 & - & + \\
\hline 19 & $23 / \mathrm{M}$ & Left FW & + & 300 & 90 & $\mathrm{~N} 1$ & 135 & 40 & 125 & - & + \\
\hline 20 & $24 / \mathrm{M}$ & Left FW & + & 325 & 80 & $\mathrm{~N} 1$ & 145 & 60 & 120 & - & + \\
\hline
\end{tabular}

Abbreviations: AP, accessory pathway; CL, cycle length; VA, ventriculoatrial conduction time (measured on the His bundle electrogram); FW, free wall; N1, normal. 
Comparative incidence of $f_{X} B B B$ at $O T$ onset with the $A_{2}$ and $V_{2}$ methods. As Table I indicates, OT was induced by the $A_{2}$ method alone in six patients (nos. 1-6), by both the $A_{2}$ and $V_{2}$ methods in seven (nos. 7-13) and by the $V_{2}$ method alone in seven (nos. 14-20).

Fig. 1 shows the distribution of widest aberrant complexes observed in each patient at OT onset, according to morphologic type and induction method. It is apparent that, with the $V_{2}$ method, not only was the incidence of $\mathrm{fx} B \mathrm{BB}$ in general greater than with the $A_{2}$ method (79\% vs. $38 \%$ ) but, more specifically, fx LBBB (complete or incomplete) was also considerably more frequent ( $79 \%$ vs. $15 \%$ with $A_{2}$ ). In contrast, fx RBBB was relatively more common at onset of OT initiated by the $A_{2}$ than by the $\mathrm{V}_{2}$ method ( $31 \%$ vs. $7 \%$ ).

Tables II and III list the actual electrophysiologic characteristics of the widest aberrant complexes (for each individual patient) at onset of OT induced by the $A_{2}$ and $V_{2}$ methods, respectively. It may be appreciated that both the predominance of $f x$ LBBB at OT onset with the $V_{2}$ method and the greater incidence of $f x R B B B$ with the $A_{2}$ method also applied to the subgroup of patients (nos. 7-13) in whom OT was inducible by both methods (with identical basic cycle lengths used in six of the seven cases). Complete RBBB and LBBB were both observed at OT onset in patient 6 with the $A_{2}$ method and in patient 9 with the $V_{2}$ method. Our electrophysiologic findings will now be considered in further detail.

Aberrant conduction at $O T$ onset with the $A_{2}$ method. As shown in Table II, five patients (nos. 5-7, 9, and 11) exhibited fx BBB at onset of OT initiated by $\mathrm{A}_{2}$, associated with an altered axis in four (nos. 6, 7, 9, and 11) and $\mathrm{HV}$ interval prolongation in all (range 10-135 ms). By the second beat of OT (not tabulated), aberrant conduction usually resolved (accompanied by shortening of the HV interval) except in two patients (nos. 6 and 11) in whom $\mathrm{fx}$ RBBB was sustained and one (no. 7) in whom infra-His block developed.

Table II also lists characteristics of the QRS complex in response to $A_{2}$ when the latter induced $\mathrm{fX} B B B$ at OT onset. Note

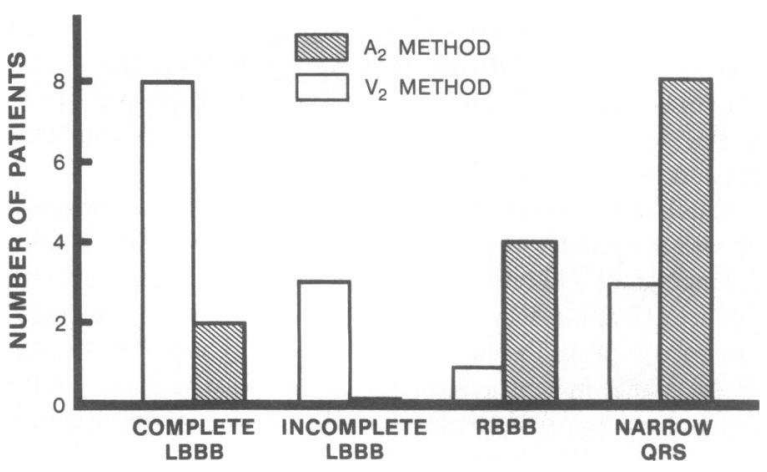

Figure 1. Comparative distribution of QRS morphologies at onset of OT induced by the $A_{2}$ and $V_{2}$ methods. Only the widest $Q R S$ complexes of a given morphology for each patient are tallied. The total number of patients here exceeds 27 (the number of those induced by $A_{2}$ plus those induced by $V_{2}$ ) because two patients had both complete RBBB and LBBB.

that in all such cases the same type of $\mathrm{fx}$ BBB occurring at OT onset was already present during conduction of $A_{2}$. In two patients (nos. 5 and 7) incomplete RBBB during propagation of $A_{2}$ progressed to complete RBBB at OT onset. Table II reveals further than the $\mathrm{HV}$ interval shortened by $10-60 \mathrm{~ms}$ during the first beat of OT (versus a longer value during the preceding complex) in five of six instances of aberrancy, with the remaining case (patient 7) manifesting progressive $\mathrm{HV}$ prolongation during fx 3:2 Wenckebach block in the His-Purkinje system $(6,10)$.

We examined a number of variables to assess their potential contribution to aberrant conduction of the $\mathrm{A}_{2}$ impulse, because the latter occurrence appeared to be a prerequisite for development of $\mathrm{fX}$ BBB during the subsequent beat (i.e., at OT onset). The ratio $\mathrm{H}_{1} \mathrm{H}_{2} / \mathrm{H}_{1} \mathrm{H}_{1}$ (where $\mathrm{H}_{1} \mathrm{H}_{1}$ = basic cycle length) was used as a measure of the degree of prematurity of a given $\mathrm{H}_{1} \mathrm{H}_{2}$ input to the His-Purkinje system. This parameter could be measured within the OT initiation zone in all patients from Table

Table II. Electrophysiologic Data in Patients with OT Induced by the $A_{2}$ Method*

\begin{tabular}{|c|c|c|c|c|c|c|c|c|c|c|c|c|c|}
\hline \multirow{2}{*}{$\begin{array}{l}\text { Patient } \\
\text { no. }\end{array}$} & \multirow[b]{2}{*}{ BCL } & \multicolumn{6}{|c|}{ Widest aberrant complex at OT onset } & \multicolumn{6}{|c|}{$\begin{array}{l}\text { Preceding complex in response to } A_{2} \text { when aberrant conduction } \\
\text { present at OT onset }\end{array}$} \\
\hline & & Morphology & $\operatorname{QRS}(\Delta)$ & Axis & HV $(\Delta)$ & $\mathrm{VA}(\Delta)$ & HH & Morphology & $\operatorname{QRS}(\Delta)$ & Axis & $\operatorname{HV}(\Delta)$ & $\mathrm{VA}(\Delta)$ & HH \\
\hline & $m s$ & & $m s$ & & $m s$ & $m s$ & $m s$ & & $m s$ & $m s$ & $m s$ & $m s$ & $m s$ \\
\hline 1 & 600 & Narrow & $80(0)$ & N1 & $50(0)$ & $110(0)$ & - & - & - & - & - & - & - \\
\hline 2 & 600 & Narrow & $90(0)$ & N1 & $50(0)$ & $125(0)$ & - & - & - & - & - & - & - \\
\hline 3 & 700 & Narrow & $80(0)$ & N1 & $55(0)$ & $120(0)$ & - & - & - & - & - & - & - \\
\hline 4 & 700 & Narrow & $70(0)$ & N1 & $55(0)$ & $150(0)$ & - & - & - & - & - & - & - \\
\hline 5 & 600 & CRBBB & $130(+50)$ & N1 & $90(+50)$ & $175(+50)$ & 330 & IRBBB & $90(+10)$ & N1 & $100(+60)$ & $140(+15)$ & 290 \\
\hline \multirow[t]{2}{*}{6} & 600 & CRBBB§ & $120(+40)$ & RAD & $95(+55)$ & $130(+10)$ & 300 & CRBBB & $120(+40)$ & N1 & $105(+65)$ & $120(0)$ & 305 \\
\hline & & CLBBB & $130(+50)$ & LAD & $50(+10)$ & $185(+65)$ & 310 & CLBBB & $130(+50)$ & LAD & $110(+70)$ & $185(+65)$ & 380 \\
\hline 7 & 600 & CRBBB & $130(+40)$ & LAD & $180(+135)$ & $160(+40)$ & 350 & IRBBB & $105(+15)$ & N1 & $130(+85)$ & $120(0)$ & 350 \\
\hline 8 & 600 & Narrow & $70(0)$ & N1 & $50(0)$ & $115(0)$ & - & - & - & - & - & - & - \\
\hline 9 & 600 & CLBBB & $135(+45)$ & LAD & $70(+20)$ & $210(+70)$ & 390 & CLBBB & $135(+45)$ & LAD & $110(+60)$ & $210(+70)$ & 410 \\
\hline 10 & 600 & Narrow & $80(0)$ & N1 & $35(0)$ & $100(0)$ & - & - & - & - & - & - & - \\
\hline 11 & 500 & CRBBBß & $120(+30)$ & RAD & $55(+10)$ & $110(0)$ & 280 & CRBBB & $120(+30)$ & N1 & $95(+50)$ & $110(0)$ & 290 \\
\hline 12 & 450 & Narrow & $80(0)$ & N1 & $40(0)$ & $120(0)$ & - & - & - & - & - & - & - \\
\hline 13 & 600 & Narrow & $90(0)$ & N1 & $40(0)$ & $120(0)$ & - & - & - & - & - & - & - \\
\hline
\end{tabular}

Abbreviations: BCL, basic cycle length; OT, orthodromic tachycardia; CRBBB, complete right bundle branch block; IRBBB, incomplete right bundle branch block; CLBBB, complete left bundle branch block; ILBBB, incomplete left bundle branch block; LAD, left axis deviation; RAD, right axis deviation. * Numbers in parentheses indicate differences from values of corresponding parameters in Table I. ¥ Refers to HH interval immediately preceding the subsequent beat; tabulated only for complexes showing aberrancy. § Sustained aberrant conduction. 
Table III. Electrophysiologic Data in Patients with OT Induced by the $V_{2}$ Method* $^{*}$

\begin{tabular}{|c|c|c|c|c|c|c|c|c|c|c|c|c|c|}
\hline \multirow{2}{*}{$\begin{array}{l}\text { Patient } \\
\text { no. }\end{array}$} & \multirow[b]{2}{*}{ BCL } & \multicolumn{6}{|c|}{ Widest aberrant complex at onset of OT initiated by $V_{2}$} & \multicolumn{6}{|c|}{ Widest aberrant complex at onset of OT initiated by $V_{3}$} \\
\hline & & Morphology & $\operatorname{QRS}(\Delta)$ & Axis & $\operatorname{HV}(\Delta)$ & VA $(\Delta)$ & HH & Morphology & QRS $(\Delta)$ & Axis & HV $(\Delta)$ & VA $(\Delta)$ & HH‡ \\
\hline & $m s$ & & $m s$ & & $m s$ & $m s$ & ms & & $m s$ & & $m s$ & ms & $m s$ \\
\hline 7 & 600 & CLBBB & $130(+40)$ & LAD & $95(+50)$ & $170(+50)$ & 370 & - & - & - & - & - & - \\
\hline 8 & 600 & ILBBB & $100(+30)$ & LAD & $60(+10)$ & $115(0)$ & 340 & - & - & - & - & - & - \\
\hline \multirow[t]{2}{*}{9} & 600 & CLBBB & $135(+45)$ & LAD & $100(+50)$ & $200(+60)$ & 410 & - & - & - & - & - & - \\
\hline & & CRBBB & $120(+30)$ & RAD & $50(0)$ & $140(0)$ & 370 & - & - & - & - & - & - \\
\hline 10 & 500 & - & - & - & - & - & - & CLBBBß & $130(+50)$ & N1 & $45(+10)$ & $100(0)$ & 310 \\
\hline 11 & 500 & - & - & - & - & - & - & CLBBBß & $150(+60)$ & N1 (L)" & $60(+15)$ & $135(+25)$ & 275 \\
\hline 12 & 450 & - & - & - & - & - & - & Narrow & $80(0)$ & N1 & $40(0)$ & $120(0)$ & 290 \\
\hline 13 & 600 & - & - & - & - & - & - & Narrow & $90(0)$ & N1 & $40(0)$ & $120(0)$ & 370 \\
\hline 14 & 600 & - & - & - & - & - & - & CLBBBß & $130(+50)$ & LAD & $70(+30)$ & $90(0)$ & 260 \\
\hline 15 & 600 & CLBBBß & $130(+50)$ & LAD & $100(+50)$ & $100(0)$ & 310 & - & - & - & - & - & - \\
\hline 16 & 700 & Narrow & $80(0)$ & N1 & $45(0)$ & $120(0)$ & 380 & - & - & - & - & - & - \\
\hline 17 & 700 & ILBBB & $90(+10)$ & LAD & $65(+15)$ & $150(+30)$ & 430 & - & - & - & - & - & - \\
\hline 18 & 700 & ILBBB & $100(+10)$ & LAD & $70(+20)$ & $140(+10)$ & 360 & - & - & - & - & - & - \\
\hline 19 & 600 & CLBBB & $150(+60)$ & LAD & $50(+10)$ & $180(+55)$ & 345 & Narrow & $90(0)$ & $\mathrm{N} 1$ & $40(0)$ & $125(0)$ & 290 \\
\hline 20 & 700 & CLBBB & $140(+60)$ & LAD & $80(+20)$ & $160(+40)$ & 360 & Narrow & $80(0)$ & N1 & $60(0)$ & $120(0)$ & 330 \\
\hline
\end{tabular}

Abbreviations are the same as in Table II. * Numbers in parenthesis indicate differences from values of corresponding parameters in Table I. $¥$ Refers to HH interval immediately preceding the subsequent beat. § Sustained aberrant conduction. "Normal axis, but more leftward than during narrow QRS OT.

II except for two (nos. 3 and 4 ) in whom the $\mathrm{H}_{1}$ deflection was obscured by a delta wave. The mean maximum $\mathrm{H}_{1} \mathrm{H}_{2} / \mathrm{H}_{1} \mathrm{H}_{1}$ value in patients who developed $\mathrm{fx}$ BBB during propagation of the $A_{2}$ impulse was smaller than the mean minimum attainable value measured in those without aberrant condition $(0.59 \pm 0.05$, range $0.52-0.64$, vs. $0.69 \pm 0.07$, range $0.59-0.78$, respectively; $P<0.02$ ). A similar relationship obtained when absolute $\mathrm{H}_{1} \mathrm{H}_{2}$ intervals were compared (i.e., mean maximum $\mathrm{H}_{1} \mathrm{H}_{2}$ in patients with aberrancy was $341 \pm 37 \mathrm{~ms}$ vs. mean minimum value of $401 \pm 63 \mathrm{~ms}$ in those without aberrancy, $P<0.05$ ).

Of note, manifest preexcitation was more common (88\%) in the group that did not develop $\mathrm{fx}$ BBB than in those patients who demonstrated aberrant conduction (only 20\%). Moreover, in all cases of manifest preexcitation the minimum $\mathrm{H}_{1} \mathrm{H}_{2}$ value associated with induction of OT was attained at the longest $A_{1} A_{2}$ coupling interval within the OT initiation zone. This interval, of course, coincided with the anterograde effective refractory period of the accessory pathway, which happened to be relatively short, ranging from 230 to $310 \mathrm{~ms}$ at the basic cycle length utilized. Finally, it should be mentioned that mean OT cycle length and mean basic cycle length (during the $A_{2}$ method) were not significantly different in the group exhibiting aberrant conduction compared to those patients who did not.

Aberrant conduction at OT onset with the $V_{2}$ method. As Table III indicates, in patients with OT induced by the $V_{2}$ method, the initiating impulse consisted of $V_{2}$ alone in seven patients (nos. 7-9 and 15-18), macro-reentrant $V_{3}$ alone in five (nos. 10-14), and either $\mathrm{V}_{2}$ or $\mathrm{V}_{3}$ (at different coupling intervals) in two (nos. 19 and 20). The site of retrograde block of the initiating impulse was determined using previously published criteria (4), and was located below the His bundle in all cases.

Aberrant conduction at OT onset was observed in 11 patients (fx LBBB in patients 7-11, 14, 15, and 17-20 and fx RBBB in patient 9). This was usually associated with axis deviation (leftward in all with $\mathrm{fx} \mathrm{LBBB}$, except for patient 10 , and rightward in the one case of $f x$ RBBB-patient 9). The HV interval pro-



Figure 2. Induction by the $\mathrm{V}_{2}$ method of $\mathrm{fx}$ sustained LBBB at OT onset in a patient (no. 10) with a paraseptal accessory pathway. Tracings from top to bottom are ECG leads $1,2, \mathrm{~V}_{1}$, high right atrium, coronary sinus, and His bundle electrograms, followed by time line. The basic cycle length $\left(S_{1} S_{1}\right.$ or $V_{1} V_{1}$ ) is 500 ms. At an $S_{1} S_{2}$ coupling interval of $260 \mathrm{~ms}$, a macro-reentrant beat $\left(\mathrm{V}_{3}\right)$ is induced $(9,11)$ which in turn retrogradely blocks bilaterally in the His-Purkinje system, permitting initiation of OT (4). Fx complete LBBB, which is observed at OT onset, sustains for another four beats before spontaneously resolving over the subsequent two beats, despite minimal changes in HH cycle length. When the QRS fully normalizes, there is associated VV shortening (because of less HV delay) and the reentrant wavefront blocks retrogradely in the accessory pathway, thereby terminating the tachycardia. It should be mentioned that the $\mathrm{VA}_{c}$ interval during sustained LBBB (100 ms, unlabeled) is identical to that measured (elsewhere) during narrow QRS OT. HRA, high right atrium; CS, coronary sinus; HB, His bundle; T, time line; A, atrial electrogram; $A_{e}$, atrial echo; $V$, ventricular electrogram; $S$, stimulus artifact. 
longed by $25 \pm 16 \mathrm{~ms}$ (range $10-50 \mathrm{~ms}$ ) in all cases of $\mathrm{fx}$ LBBB, but remained unchanged in the single instance of $f x$ RBBB. Sustained fx LBBB beginning at OT onset was observed in four patients (nos. 10,11,14, and 15). An example from patient 10, in whom sustained $\mathrm{LBBB}$ resolved spontaneously after several beats, is shown in Fig. 2.

Retrograde concealed conduction in the His-Purkinje system at onset of OT induced by the $V_{2}$ method. In patients with OT induced by the $V_{2}$ method, we sought to determine whether extent of His-Purkinje system recovery, as a function of elapsed time after concealed retrograde impulse penetration, could play a role in the genesis of $f x$ BBB at OT onset. The possible existence of such a relationship was investigated by plotting the ventricular-His (VH) intervals corresponding to the widest initial OT complexes for each patient in whom OT was induced by the $V_{2}$ method (excluding the single case of $f x$ RBBB, discussed separately below).

The resulting graph is shown in Fig. 3. Note that the VH interval for narrow initial QRS complexes (351 $\pm 42 \mathrm{~ms})$ was significantly greater $(P<0.01)$ than the value of that parameter determined when either $\mathrm{fx}$ incomplete or complete LBBB was manifest at OT onset ( $262 \pm 18$ and $238 \pm 43 \mathrm{~ms}$, respectively). It follows from Table III that the HV interval at OT onset varied reciprocally with $\mathrm{VH}$ because the $\mathrm{HV}$ interval increased from $45 \pm 9$ to $65 \pm 5$ to $75 \pm 22 \mathrm{~ms}$ for narrow $\mathrm{QRS}$, incomplete, and complete LBBB, respectively.

The relationship between $\mathrm{VH}$ interval and occurrence of $f x$ LBBB was also examined within OT initiation zones of eight individual patients in whom OT was induced at more than one $\mathrm{S}_{1} \mathrm{~S}_{2}$ coupling interval. In these cases the corresponding "VH zones" spanned a width ranging from 10 to $40 \mathrm{~ms}$. In four of five patients manifesting complete LBBB at OT onset, this widest aberrantly conducted complex occurred at the lower limit of the VH zone, whereas fx incomplete LBBB or a narrow QRS complex was observed at the longest $\mathrm{VH}$ interval. An example of this relationship is shown in Fig. 4, taken from patient 9. Note the progression at OT onset in $A-C$ from narrow $\mathrm{QRS}$ to incomplete and then complete fx LBBB (with associated progressive $\mathrm{HV}$ prolongation) as the $\mathrm{VH}$ interval decreases from longest to shortest value.

The single case of $\mathrm{fx}$ RBBB, which we observed (in patient 9) at the onset of OT initiated by $V_{2}$, deserves special mention. As shown in Fig. $5 \mathrm{~A}$, the His-right bundle temporal relationship during fx RBBB is identical to that existing during OT. This finding confirms that the aberrantly appearing complex is truly of anterograde origin, rather than representing atypical bundle branch reentry $(9,11,13)$, as indeed occurred in this patient when the $S_{1} S_{2}$ coupling interval was decreased by 10 ms (Fig. 5 $B)$. The relationship between $\mathrm{RBBB}$ at OT onset and retrograde concealed conduction in the His-Purkinje system will be considered below in the Discussion.

Effect of aberrant conduction at OT onset upon the VA interval. In this study we were able to assess specifically the effect of aberrant conduction during a single beat (i.e., at OT onset) upon the VA interval. Complete BBB ipsilateral to a free wall accessory pathway was observed at OT onset in six patients (nos. $5-7,9,19$, and 20). In these cases the associated (initial) VA interval was $55 \pm 11 \mathrm{~ms}$ (range 40-70 ms) greater than the corresponding value during narrow $\mathrm{QRS} O \mathrm{OT}$. Such prolonged VA intervals at OT onset fell within $10 \mathrm{~ms}$ (mean $-3 \pm 4 \mathrm{~ms}$ ) of the values measured in five of the six patients during sustained ipsilateral $\mathrm{fx}$ BBB.

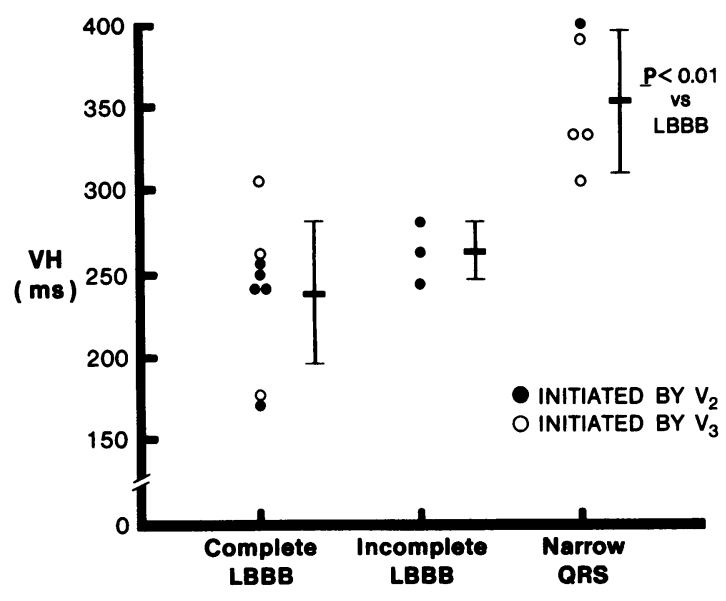

Figure 3. Plot of $\mathrm{VH}$ (i.e., $\mathrm{V}_{2} \mathrm{H}$ or $\mathrm{V}_{3} \mathrm{H}$ ) intervals corresponding to morphology of widest $\mathrm{QRS}$ complexes at onset of OT initiated by the $V_{2}$ method. The total number of points plotted exceeds 14 (the number of patients with OT induced by the $V_{2}$ method) because in two cases OT could be initiated by either $\mathrm{V}_{2}$ or $\mathrm{V}_{3}$. The $P$ value shown corresponds to the difference in $\mathrm{VH}$ for narrow $\mathrm{QRS}$ versus either complete or incomplete LBBB.

We considered the possibility that initial VA prolongation might have resulted in part from an incompletely recovered bypass tract rather than simply from delayed retrograde input to that tissue (owing to ipsilateral BBB). Such an interpretation appears unlikely inasmuch as the retrograde coupling interval $\left(\mathrm{V}_{2} \mathrm{~V}\right.$ or $\left.\mathrm{V}_{3} \mathrm{~V}\right)$ associated with $\mathrm{fx}$ complete BBB at OT onset greatly exceeded the retrograde effective refractory period of the accessory pathway, namely, by $65 \pm 29 \mathrm{~ms}$ (range $30-110 \mathrm{~ms}$ ). This point may be illustrated (in patient 9) by comparing the $\mathrm{V}_{2} \mathrm{~V}$ interval in Fig. $3 C$ (corresponding to complete LBBB) with the $V_{1} V_{2}$ interval in Fig. $4 B$ (where the retrograde effective refractory period of the bypass tract is encountered).

At OT onset, fx complete BBB contralateral to a free wall bypass tract was observed in three patients (nos. 6, 7, and 9). The associated VA interval was prolonged by no more than 10 ms, except in one (patient 7) who exhibited fx bifascicular block (at onset of OT induced by $A_{2}$ ). Of the patients with a paraseptal pathway, both $\mathrm{fx}$ complete RBBB and LBBB occurred at OT onset in one (no. 11). In that case, the VA interval increased solely during complete LBBB (by $25 \mathrm{~ms}$, an increment identical to that observed during sustained LBBB).

\section{Discussion}

Our results suggest that in patients with Wolff-Parkinson-White syndrome, the $A_{2}$ and $V_{2}$ methods each tend to promote specific types of aberrant conduction at onset of induced OT. In the series of patients that we studied, fx LBBB was readily induced by the $V_{2}$ method, whereas $f x$ RBBB was more frequent with the $A_{2}$ method. Moreover, the overall incidence of aberrant conduction was greater with the $V_{2}$ method. Because random variation in patient selection and use of different basic cycle lengths can alter the actual incidences of particular types of aberrant conduction from one clinical series to another, we wish to place greater emphasis on the roles played by differences in electrophysiologic sequelae of the $A_{2}$ and $V_{2}$ methods.

Relation of the OT initiating mechanism with the $A_{2}$ method 


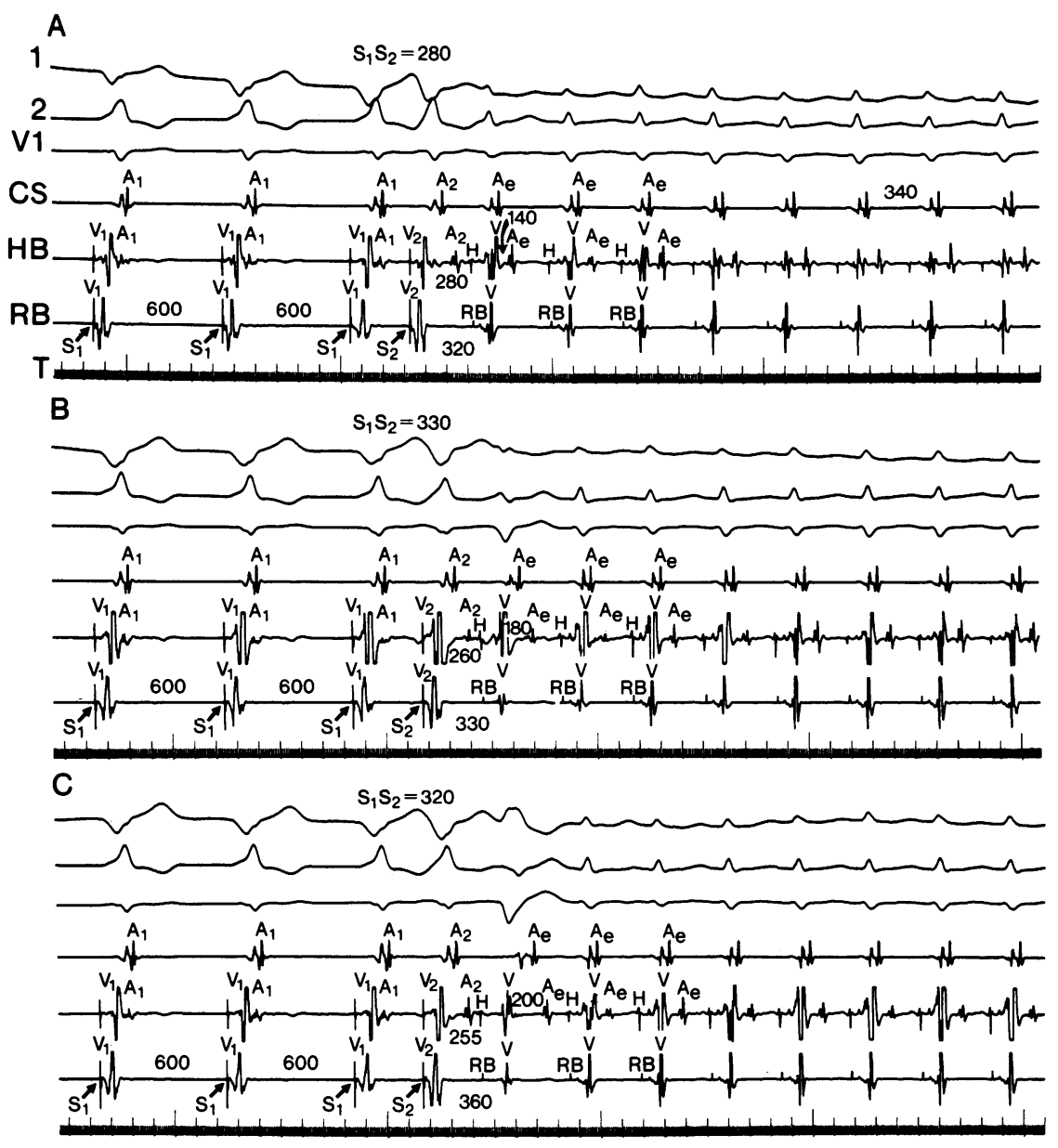

Figure 4. Induction of $\mathrm{fx}$ LBBB at onset of OT initiated by the $V_{2}$ method in a patient (no. 9) with a concealed left free wall accessory pathway. Tracings from top to bottom are ECG leads 1,2 , and $V_{1}$ followed by coronary sinus, His bundle and right bundle electrograms, and time line. In all three panels a ventricular extrastimulus $\left(S_{2}\right.$ or $\left.V_{2}\right)$ initiates OT after the last beat of the basic ventricular drive which has a cycle length $\left(S_{1} S_{1}\right.$ or $\left.V_{1} V_{1}\right)$ of $600 \mathrm{~ms}$. The panels are arranged in order of increasing $\mathrm{V}_{2} \mathrm{H}$ intervals. (A) At an $\mathrm{S}_{1} \mathrm{~S}_{2}$ (or $V_{1} V_{2}$ ) coupling interval of $280 \mathrm{~ms}$, the $V_{2}$ impulse initiates an OT with narrow initial QRS complex. This beat is associated with a $\mathrm{V}_{2} \mathrm{H}$ interval of $280 \mathrm{~ms}$ and a VA $\mathrm{VA}_{e}$ interval of 140 ms. The HV and RB-V intervals at OT onset (45 and $25 \mathrm{~ms}$, respectively, unlabeled) are identical to the values measured during sustained narrow QRS OT at a cycle length of $340 \mathrm{~ms}$. Subtle QRS alternans, which may occur during OT in patients with Wolff-Parkinson-White syndrome (12), is also noted here. (B) At a longer $S_{1} S_{2}$ coupling interval of $330 \mathrm{~ms}$ OT is again initiated, but now $\mathrm{x}$ incomplete LBBB is observed at OT onset. This occurs in association with a shorter $\mathrm{V}_{2} \mathrm{H}$ interval of $260 \mathrm{~ms}$ and an increase in both the $\mathrm{HV}$ and RB-V intervals to 80 and $60 \mathrm{~ms}$, respectively (both unlabeled). The initial $\mathrm{VA}_{e}$ interval also prolongs to $180 \mathrm{~ms}$. $(C)$ At a slightly shorter $S_{1} S_{2}$ coupling interval of 320 $\mathrm{ms}$, $\mathrm{fx}$ complete LBBB is now apparent at OT onset whereas the $\mathrm{V}_{2} \mathrm{H}$ interval has shortened further (versus $B$ ) to $255 \mathrm{~ms}$. Concomitantly,

the $\mathrm{VA}_{\mathrm{e}}$ interval prolongs to $200 \mathrm{~ms}$, a value only $10 \mathrm{~ms}$ less than that measured (elsewhere) during sustained fx LBBB. The initial HV and RB-V intervals have also increased to $100 \mathrm{~ms}$ and $80 \mathrm{~ms}$, respectively (unilabeled). Preservation of the His-RB relationship that exists during narrow QRS OT ensures that the wide QRS complex at OT onset is of anterograde origin (and argues against the occurrence of a macro-reentrant $V_{3}$ ) (11). Moreover, the fact that in $B$ and $C$ both the HV and RB-V prolong by the same amount implies that, at least in this case, the site of coexistent conduction delay along the H-RB-V route is located distal to the RB recording site, presumably where retrograde concealed penetration of the $R B$ by the $V_{2}$ impulse had occurred (13). This state of affairs is different from that which obtains during fx BBB induced by atrial premature stimulation where the site of associated right-sided conduction delay is usually located proximal to the $\mathrm{RB}$ recording site (14). It should also be evident from $A-C$ that at the same time that greater degrees of $\mathrm{fx}$ LBBB are induced at OT onset, the $\mathrm{V}_{2} \mathrm{~V}$ interval progressively increases from 320 to $360 \mathrm{~ms}$, making it most unlikely that the concomitant progressive $\mathrm{VA}_{e}$ prolongation can be attributed to greater retrograde input stress on the accessory pathway. Finally, it may be appreciated in $C$ that fx complete LBBB at OT onset resolves by the second beat of OT in association with marked $\mathrm{HH}$ prolongation (to $420 \mathrm{~ms}$, unlabeled) despite AH shortening relative to narrow QRS OT. RB, right bundle; other abbreviations as in Fig. 2.

to aberrant conduction at OT onset. For OT to be initiated by an atrial extrastimulus, a critical amount of anterograde delay in transmission of the $A_{2}$ impulse is required to permit recovery of the bypass tract (from the effects of concealed anterograde penetration) so that reentrant excitation of the atria can occur (3). To this end, slowing of impulse propagation solely in the atrioventricular node, at a sufficiently short $A_{1} A_{2}$ coupling interval, is often adequate $(3,6)$. Less commonly additional delays in anterograde His-Purkinje system conduction and/or disruption of the normal ventricular activation sequence are needed, as may be afforded by the occurrence of fx BBB, especially the ipsilateral type $(6,15,16)$.

Whether or not $\mathrm{fx}$ BBB is a necessary condition for OT initiation by the $A_{2}$ method in a given patient, the occurrence of aberrant conduction will have similar determinants in all cases. Most important of these is the ability of the atrioventricular node, over a range of $A_{1} A_{2}$ coupling intervals, to deliver suff- ciently short $\mathrm{H}_{1} \mathrm{H}_{2}$ inputs that will encroach upon the relative refractory period of the His-Purkinje system (17). In our cases of OT induced by the $\mathrm{A}_{2}$ method, shorter $\mathrm{H}_{1} \mathrm{H}_{2}$ intervals (both absolute and relative to basic cycle length) were indeed found in patients who exhibited $\mathrm{fx} B \mathrm{BB}$ during propagation of $\mathrm{A}_{2}$ compared to those who did not.

Notably, we also found that a relatively short anterograde effective refractory period of the accessory pathway appeared to be an indirect (but potentially important) factor limiting the ocurrence of aberrant conduction during transmission of $\mathrm{A}_{2}$ impulses that initiated OT. Such an impression is based upon observations in our patients with manifest preexcitation, all of whom had short anterograde accessory pathway refractory periods and most of whom did not exhibit fx BBB after $A_{2}$. In these cases, the minimum $\mathrm{H}_{1} \mathrm{H}_{2}$ interval associated with OT induction was found at the longest $A_{1} A_{2}$ coupling interval within the OT initiation zone. This finding will require confirmation 


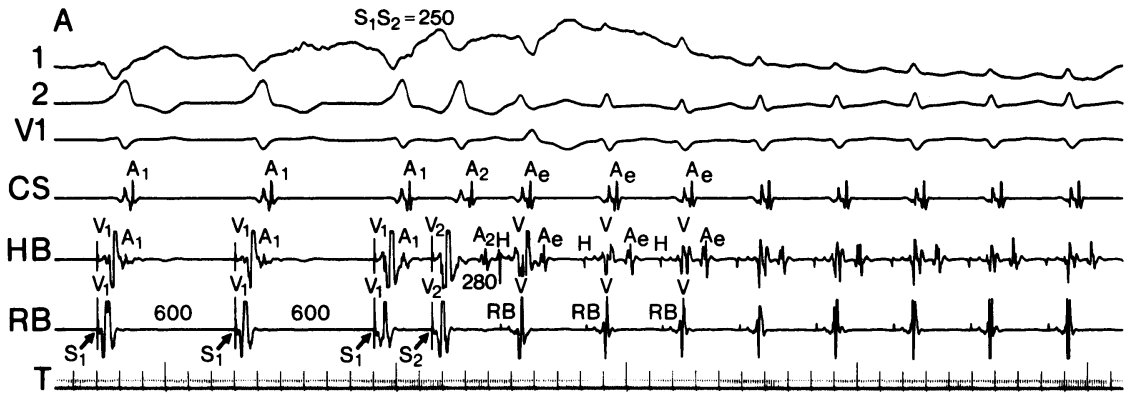

B

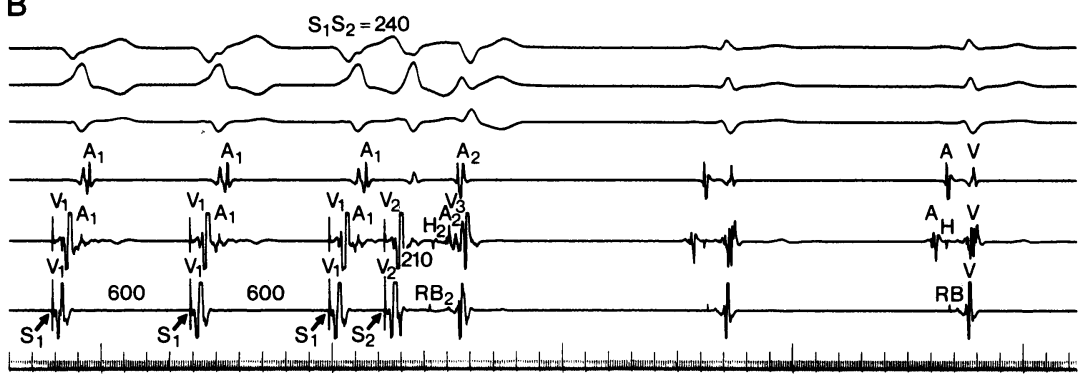

Figure 5. Induction of $\mathrm{fx}$ RBBB at onset of OT initiated by the $V_{2}$ method (same patient as in Fig. 4). Format, abbreviations, and basic cycle length are identical to those of Fig. 4. (A) At an $\mathrm{S}_{1} \mathrm{~S}_{2}$ coupling interval of $250 \mathrm{~ms}$ ( 30 ms shorter than in Fig. $4 \mathrm{~A}$ ) complete RBBB at onset of OT occurs with a $\mathrm{V}_{2} \mathrm{H}$ interval of $280 \mathrm{~ms}$ and unaltered $\mathrm{HV}$ and RB$\mathrm{V}$ intervals compared to narrow QRS OT (45 and $25 \mathrm{~ms}$, respectively, unlabeled). The preserved His-RB relationship is consistent with a supraventricular site of impulse origin (and block in the $R B$ below the $R B$ recording site). (B) When $\mathrm{S}_{1} \mathrm{~S}_{2}$ is shortened to $240 \mathrm{~ms}$, the retrograde effective refractory period of the accessory pathway is encountered and macroreentry with a RBBB morphology ensues (9, 11). The latter phenomenon is associated with a resumption of retrograde conduction over the normal pathway despite bilateral His-Purkinje system block in $A$ (gap phenomenon) (13). That His activation occurs retrogradely here (versus anterogradely in $A$ )

follows from postgap shortening of the $\mathrm{V}_{2} \mathrm{H}$ interval (4) in $B$ (to $210 \mathrm{~ms}$ ) and from the alteration in the His- $\mathrm{RB}$ relationship $(11,13)$ so that the $\mathrm{RB}$ deflection now precedes the $\mathrm{H}$ by $20 \mathrm{~ms}$. This clearly differs from the situation during narrow QRS OT (in $A$ ) or during sinus beats (in $B$ ). Finally, note that in contrast to the case of fx complete LBBB at OT onset (Fig. $4 C$ ), there is no VA prolongation (relative to narrow QRS OT) associated with complete $\mathrm{RBBB}$ at OT onset $\left(\mathrm{VA}_{e}=140 \mathrm{~ms}\right.$, unlabeled in $\left.B\right)$ in this patient with a left free wall accessory pathway.

in a larger series. Nonetheless, it implies that the atrioventricular nodal functional refractory period (and, hence, shorter $\mathrm{H}_{1} \mathrm{H}_{2}$ values) would have been encountered only at $A_{1} A_{2}$ coupling intervals exceeding the anterograde effective refractory period of the bypass tract (i.e., beyond the OT initiation zone).

When $f x$ BBB did occur during propagation of $A_{2}$, we observed that the same type of aberrant conduction was sustained through the next beat (first OT complex), presumably through a linking-by-interference mechanism (18) involving transseptal retrograde invasion from the contralateral bundle (19). Because the $A_{2}$ method typically induces $f x$ RBBB more commonly than fx LBBB (17), it is not surprising that such was also the case in our series during conduction of $\mathrm{A}_{2}$ and, hence, at OT onset.

Relation of the OT initiating mechanism with the $V_{2}$ method to aberrant conduction at OT onset. The mechanism of OT initiation with the $\mathrm{V}_{2}$ method is fundamentally different from that of its atrial counterpart. As depicted schematically in Fig. 6, during the basic drive $\left(\mathrm{V}_{1}\right.$, upper left corner) both the normal and the accessory pathways are activated retrogradely. We (4) have shown previously that in order for OT to be initiated, in most instances bilateral retrograde infra-His (rather than intranodal) block of the $\mathrm{V}_{2}$ impulse (Fig. 6, upper middle panel) or the macro-reentrant $\mathrm{V}_{3}$ impulse (Fig. 6, lower left and middle panels) must occur, with retrograde atrial activation proceeding exclusively via the accessory pathway. In either case, because ventricular activation during $V_{2}$ and $V_{3}$ (of the typical variety $[9,11])$ originates from a right ventricular location, concealed retrograde penetration of the left bundle will be delayed relative to occurrence of similar concealment in the right bundle. Consequently, recovery of excitability in the left bundle will be completed at a later time than in the case of its right-sided counterpart.

In virtually all patients, therefore, a bias exists that clearly favors the development of $\mathrm{fx}$ LBBB when the initial reentrant impulse attempts to traverse the His-Purkinje system. More precisely, anterograde engagement of the left bundle will occur during its recovery process at a point determined by the arrival time of the anterograde impulse to the His-Purkinje system relative to the preceding retrograde penetration (i.e., the $\mathrm{VH}$ interval). This arrival time, in turn, is largely a function of anterograde atrioventricular nodal conduction (since VH = VA $+\mathrm{AH}$, and VA is virtually fixed in any given patient).



Figure 6. Schematic representation of mechanism responsible for facilitation of $f x$ LBBB at onset of OT induced by the $V_{2}$ method. Paced stimuli (asterisks) that give rise to $V_{1}$ and $V_{2}$ are delivered to a right ventricular site. The location of the accessory pathway is arbitrary. Moreover, depicted sites of conduction delay or block in the His-Purkinje system (shaded regions) are purely schematic in nature and not intended to imply precise anatomic locations. See text for additional explanation. AVN, atrioventricular node; $\mathrm{AP}$, accessory pathway; HB, His bundle; RB, right bundle; LB, left bundle; VM, ventricular myocardium; Ve, first ventricular echo of OT. 
We were able to demonstrate that indeed the magnitude of the $\mathrm{VH}$ interval could be correlated with the occurrence of $\mathrm{fx}$ LBBB at onset of OT induced by the $V_{2}$ method. Over the range of basic cycle lengths utilized, fx LBBB was observed only when VH approached the vicinity of $300 \mathrm{~ms}$ or less, with complete (rather than incomplete) LBBB observed at the shortest VH intervals (Fig. 3). The reciprocal VH-HV relationship that we documented implies encroachment upon the relative refractory period of the right bundle, as well as left bundle, when the VH interval is short (Fig. 4). Ultimately, if VH were sufficiently short, bilateral infra-His block of the anterograde impulse would occur $(5,18)$.

Although not immediately apparent, it is possible to account also for the much less likely occurrence of $\mathrm{fx}$ RBBB at onset of OT induced with $\mathrm{V}_{2}$ (Fig. $5 \mathrm{~A}$ ) by invoking a mechanism analogous to that which appears responsible for the genesis of $f x$ LBBB, as follows: at short coupling intervals during the $V_{2}$ technique, retrograde His activation usually occurs via the transseptal-left bundle route (with retrograde right bundle conduction either blocked or markedly slowed) (13). In a small subset of patients, however, the opposite situation may obtain at certain short coupling intervals so that retrograde block (or slow conduction) occurs in the left bundle while retrograde His activation proceeds via the right bundle route (13).

Under appropriate conditions this scenario could lead to macro-reentry with a RBBB morphology (11), as indeed was the case in Fig. 5 B. By extrapolation, in some instances of bilateral infra-His block of the $\mathrm{V}_{2}$ impulse, retrograde block (and recovery) in the left bundle might actually precede occurrence of retrograde block (and recovery) in the right bundle. Hence, if a patient with a retrogradely functioning accessory pathway developed this type of bilateral retrograde His-Purkinje system block during the $-\mathbf{V}_{\mathbf{2}}$ method, then fx R (not L) BBB would be facilitated at OT onset, as in Fig. $5 \mathrm{~A}$.

Clinical implications. Our findings can be applied to the development of a rational approach to the deliberate induction of specific types of $\mathrm{fx}$ BBB using programmed stimulation in patients with Wolff-Parkinson-White Syndrome. Because aberrant conduction at onset of OT induced by the $A_{2}$ method appears largely determined by the occurrence (and type) of $\mathrm{fx}$ BBB during anterograde propagation of $A_{2}$, techniques that increase the "yield" of fx (usualy R) BBB during conduction of $A_{2}$ should also facilitate occurrence of (R)BBB at OT onset. Such techniques might include not only use of both long and short basic cycle lengths (the latter being perhaps more likely to promote fx LBBB [20]) but also maneuvers that prolong His-Purkinje system refractoriness, such as abrupt short-to-long cycle length changes (21) and bigeminal rhythms (22).

Because occurrence of bilateral retrograde His-Purkinje system block during the $\mathrm{V}_{2}$ method permits initiation of OT at the same time that it facilitates $\mathrm{fx}$ LBBB at OT onset, all attempts to induce OT by this technique should be made. As in the case of the $\mathrm{A}_{2}$ method, ventricular drives incorporating short-to-long cycle-length changes (23) or alternating long and short cycle lengths (24) might prove helpful. Bilateral fx retrograde HisPurkinje system block can also often be induced by a ventricular train consisting of two short cycles of equal duration (25). This technique can result in OT initiation (26) and facilitates fx LBBB at OT onset (18) by a mechanism similar to that which is operative with the $V_{2}$ method. If OT with only a narrow initial QRS is induced by the aforementioned ventricular stimulation techniques, then the VH interval is probably not sufficiently short. This situation might be remedied by administration of an agent such as atropine, which enhances atrioventricular nodal conduction (27).

Our findings also provide a mechanistic rationale for proposing a novel approach to the deliberate induction of $f x$ RBBB at OT onset. By performing ventricular extrastimulation from the left ventricle, a mirror image of the events depicted schematically in Fig. 6 might very well obtain, so that at OT onset a bias would then exist favoring the occurrence of fx RBBB.

Finally, our data suggest that quantitation of changes in the VA interval associated with $f x$ BBB at OT onset alone may aid in accessory pathway localization. This underscores the importance of being able deliberately to induce even a single aberrantly conducted beat at the onset of OT.

\section{Acknowledgment}

The authors thank Barbara O'Leary and Brian Miller for their assistance in the preparation of this manuscript.

\section{References}

1. Coumel, P., and P. Attuel. 1974. Reciprocating tachycardia in overt and latent preexcitation: influence of bundle branch block on the rate of the tachycardia. Eur. J. Cardiol. 1:423-436.

2. Kerr, C. R., J. J. Gallagher, and L. D. German. 1982. Changes in ventriculoatrial intervals with bundle branch block aberration during reciprocating tachycardia in patients with accessory atrioventricular pathways. Circulation. 66:196-201.

3. Durrer, D., L. Shoo, R. M. Schuilenburg, and H. J. J. Wellens. 1967. The role of premature beats in the initiation and termination of supraventricular tachycardia in the Wolff-Parkinson-White syndrome. Circulation. 36:644-662.

4. Akhtar, M., M. Shenasa, and D. H. Schmidt. 1981. Role of retrograde His Purkinje block in the initiation of supraventricular tachycardia by ventricular premature stimulation in the Wolff-Parkinson-White syndrome. J. Clin. Invest. 67:1047-1055.

5. Dongas, J., H. Charleson, M. Lehmann, R. Mahmud, S. Denker, and M. Akhtar. 1983. Electrophysiologic manifestations of retrograde concealed conduction in the human His-Purkinje system. Circulation. 68(Suppl. III):III-424. (Abstr.)

6. Akhtar, M., A. N. Damato, J. N. Ruskin, W. P. Batsford, C. P. Reddy, A. R. Ticzon, M. S. Dhatt, J. A. C. Gomes, and A. H. Calon. 1978. Antegrade and retrograde conduction characteristics in three patterns of paroxysmal atrioventricular junctional reentrant tachycardia. Am. Heart J. 95:22-42.

7. Gallagher, J. J., E. L. C. Pritchett, W. C. Sealy, J. Kasell, and A. G. Wallace. 1978. The preexcitation syndromes. Prog. Cardiovasc. Dis. 20:285-327.

8. Criteria Committee of the New York Heart Association. 1973. Diseases of the Heart and Blood Vessels. Nomenclature and Criteria for Diagnosis. Little, Brown \& Co., Boston. 238-242.

9. Akhtar, M., A. N. Damato, W. P. Batsford, J. N. Ruskin, J. B. Ogunkelu, and G. Vargas. 1974. Demonstration of re-entry within the His-Purkinje system in man. Circulation. 50:1150-1162.

10. Ross, D. L., J. Farre, F. W. H. M. Bar, E. J. Vanagt, P. Brugada, I. Wiener, and H. J. J. Wellens. 1981. Spontaneous termination of circus movement tachycardia using an accessory pathway: incidence, site of block and mechanisms. Circulation. 63:1129-1139.

11. Akhtar, M., C. Gilbert, F. G. Wolf, and D. H. Schmidt. 1978. Reentry within the His-Purkinje system: elucidation of reentrant circuit using right bundle branch and His bundle recordings. Circulation. 58: 295-304.

12. Green, M., B. Heddle, W. Dassen, M. Wehr, H. Abdollah, P. Brugada, and H. J. J. Wellens. 1983. Value of QRS alternation in de- 
termining the site of origin of narrow QRS supraventricular tachycardia. Circulation. 68:368-373.

13. Akhtar, M., C. J. Gilbert, F. G. Wolf, and D. H. Schmidt. 1979. Retrograde conduction in the His-Purkinje system: an analysis of routes of impulse propagation using His and right bundle branch recordings. Circulation. 59:1252-1265.

14. Akhtar, M., C. Gilbert, M. Al-Nouri, and S. Denker. 1980. Site of conduction delay during functional block in the His-Purkinje system in man. Circulation. 61:1239-1248.

15. Zipes, D. P., R. L. DeJoseph, and D. Rothbaum. 1974. Unusual properties of accessory pathways. Circulation. 49:1200-1211.

16. Pritchett, E. L. C., J. J. Gallagher, M. Scheinman, and W. M. Smith. 1978. Determinants of antegrade echo zone in the Wolff-Parkinson-White syndrome. Circulation. 57:671-677.

17. Denker, S. T., C. J. Gilbert, M. Shenasa, and M. Akhtar. 1983. An electrocardiographic-electrophysiologic correlation of aberrant ventricular conduction in man. J. Electrocardiol. 16:269-277.

18. Lehmann, M. H., S. Denker, R. Mahmud, A. Addas, and M. Akhtar. 1985. Linking: a dynamic electrophysiologic phenomenon in macro-reentry circuits. Circulation. 71:254-265.

19. Moe, G. K., C. Mendez, and J. Han. 1965. Aberrant A-V impulse propagation in the dog heart: a study of functional bundle branch block. Circ. Res. 16:261-286.

20. Chilson, D. A., D. P. Zipes, J. J. Heger, K. F. Browne, and E. N. Prystowsky. 1984. Functional bundle branch block: discordant response of right and left bundle branches to changes in heart rate. Am. J. Cardiol. 54:313-316.

21. Denker, S., M. Shenasa, C. J. Gilbert, and M. Akhtar. 1983. Effect of abrupt changes in cycle length on refractoriness of the HisPurkinje system in man. Circulation. 67:60-68.

22. Denker, S., M. Lehmann, R. Mahmud, C. Gilbert, and M. Akhtar. 1984. Effects of alternating cycle lengths on refractoriness of the HisPurkinje system. J. Clin. Invest. 74:559-570.

23. Denker, S., M. H. Lehmann, R. Mahmud, C. Gilbert, and M. Akhtar. 1983. Divergence between His-Purkinje system and ventricular muscle refractoriness with abrupt changes in cycle length. Circulation. 68:1212-1221.

24. Lehmann, M. H., S. Denker, R. Mahmud, and M. Akhtar. 1984. Postextrasystolic alterations in refractoriness of the His-Purkinje system and ventricular myocardium in man. Circulation. 69:1096-1102.

25. Lehmann, M. H., S. Denker, R. Mahmud, and M. Akhtar. 1983. Functional His-Purkinje system behavior during sudden ventricular rate acceleration in man. Circulation. 68:767-775.

26. Charleson, H., A. Estrada, S. Denker, M. Lehmann, R. Mahmud, and M. Akhtar. 1983. Mechanisms of orthodromic tachycardia initiation during incremental ventricular pacing in Wolff-Parkinson-White syndrome. Circulation. 68(Suppl. III):III-10. (Abstr.)

27. Akhtar, M., A. N. Damato, A. R. Caracta, W. P. Batsford, M. E. Josephson, and S. H. Lau. 1974. Electrophysiologic effects of atropine on atrio-ventricular conduction studied by His bundle electrogram. Am. J. Cardiol. 33:333-343. 